See discussions, stats, and author profiles for this publication at: https://www.researchgate.net/publication/350783313

\title{
Investigating Refractoriness in Collision Perception Neuronal Model
}

Conference Paper · July 2021

CITATIONS

0

4 authors, including:

2. Mu Hua

1 University of Lincoln

3 PUBLICATIONS 0 CITATIONS

SEE PROFILE

Shigang Yue

University of Lincoln

220 PUBLICATIONS 2,444 CITATIONS

SEE PROFILE

Some of the authors of this publication are also working on these related projects:

EU H2020 STEP2DYNA View project

Project High speed transceiver and High speed ADC View project
READS

52

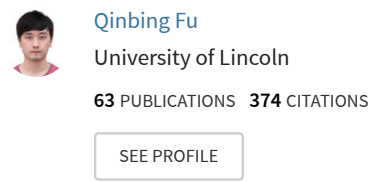




\section{Investigating Refractoriness in Collision Perception Neuronal Model}

\author{
$\mathrm{Mu}$ Hua \\ School of Computer Science \\ University of Lincoln \\ Lincoln, LN6 7TS, UK \\ mhua@lincoln.ac.uk
}

\author{
Qinbing Fu \\ School of Computer Science \\ University of Lincoln \\ Lincoln, LN6 7TS, UK \\ qinbingfu87@gmail.com
}

\author{
Wenting Duan \\ School of Computer Science \\ University of Lincoln \\ Lincoln, LN6 7TS, UK \\ wduan@lincoln.ac.uk
}

\author{
Shigang Yue \\ School of Computer Science \\ University of Lincoln \\ Lincoln, LN6 7TS, UK \\ syue@lincoln.ac.uk
}

\begin{abstract}
Currently, collision detection methods based on visual cues are still challenged by several factors including ultrafast approaching velocity and noisy signal. Taking inspiration from nature, though the computational models of lobula giant movement detectors (LGMDs) in locust's visual pathways have demonstrated positive impacts on addressing these problems, there remains potential for improvement. In this paper, we propose a novel method mimicking neuronal refractoriness, i.e. the refractory period (RP), and further investigate its functionality and efficacy in the classic LGMD neural network model for collision perception. Compared with previous works, the two phases constructing RP, namely the absolute refractory period (ARP) and relative refractory period (RRP) are computationally implemented through a 'link (L) layer' located between the photoreceptor and the excitation layers to realise the dynamic characteristic of $R P$ in discrete time domain. The $L$ layer, consisting of local time-varying thresholds, represents a sort of mechanism that allows photoreceptors to be activated individually and selectively by comparing the intensity of each photoreceptor to its corresponding local threshold established by its last output. More specifically, while the local threshold can merely be augmented by larger output, it shrinks exponentially over time. Our experimental outcomes show that, to some extent, the investigated mechanism not only enhances the LGMD model in terms of reliability and stability when faced with ultra-fast approaching objects, but also improves its performance against visual stimuli polluted by Gaussian or Salt-Pepper noise. This research demonstrates the modelling of refractoriness is effective in collision perception neuronal models, and promising to address the aforementioned collision detection challenges.
\end{abstract}

\section{INTRODUCTION}

Accurate and reliable collision detection plays a fairly crucial role for most insects. Locusts, benefiting from thousands of decades' evolution, have been equipped with a vision system which improves their success rate of evading from their natural predators coming in the blink of an eye. Within this relatively short period of time, several sets of neurons, namely the LGMDs, which respond selectively to targets on a collision course, and the descending contralateral movement detectors (DCMDs) to pass neuronal spikes to trigger evasive glide, collaborate closely to achieve avoidance [1]. Hence, upon the LGMDs, models have been established for quick collision detection, which have demonstrated reliability and robustness. Compared to conventional and complex methods such as expansion segmentation [2], these bio-plausible models, inspired from and built upon solid prior knowledges of neuroscience, have their own superiority. Not only have they taken advantages of reliable conclusions summed by neurobiologists, it is also their being low in algorithm complexity that makes it possible to be integrated with low energy-consuming platforms, Colias, for instance [3].

However, with respect of all these pros that bio-plausible neural networks are distinguished by, they can be deficient in certain aspects. In terms of robustness and accuracy, there is no doubt that convolutional neural networks of lane/vehicle detection and ROI (Region Of Interest) are there to be caught up with [4]. Multiple methods that require various sorts of hardware have also been proposed for solving the collision detection problem. For example, in [5], they propose a method based on visual cue captured and processed by one onboard camera together with a high-performance GPU, succeeding in detecting long distance collision (400 metres to 900 metres) and accordingly sending out warnings. Fusion of sensors for collision perception and drivable tunnel recreation has also been realized by Nedevschi et al., utilizing stereo vision [6]. It as well detects pending collision quickly at acceptable success rate. Thus, in what way improvement on bio-plausible methods' performance and reliability can be achieved when the velocity of objects on approaching trajectory is beyond fast, as well as when input signals are noise-polluted, remains to be explored.

Refractoriness, known also as Refractory Period (RP) in both invertebrates' and vertebrates' neural system, is a common phenomenon. Caused by inactivation of sodium channels that help depolarize the membrane, one entire process of RP is divided into ARP and RRP as shown in Fig. 1(a). Within the short period of time of ARP, neurons remain completely silent while during RRP, they retain the possibility of producing spikes again for stronger stimuli [7].

The principles of RP, plus mechanism, has been being under researched since 1950s. Although RP has been mentioned as a phenomenon in literature, hardly is it considered in modelling for locusts' visual system, not to mention in modelling for LGMD for collision perception. Focused on filling the gap, in the attempt to model RP mechanism for discrete signals, comparative experiments have been conducted in a systematic manner. From the collected consequences, our model shows, this mechanism assists the classic LGMD model with de- 


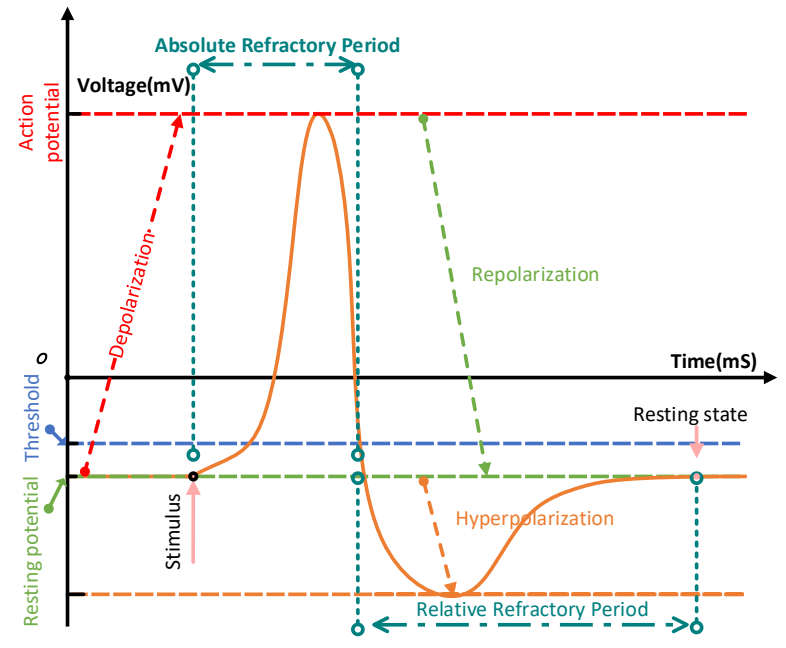

(a) Refractory Period

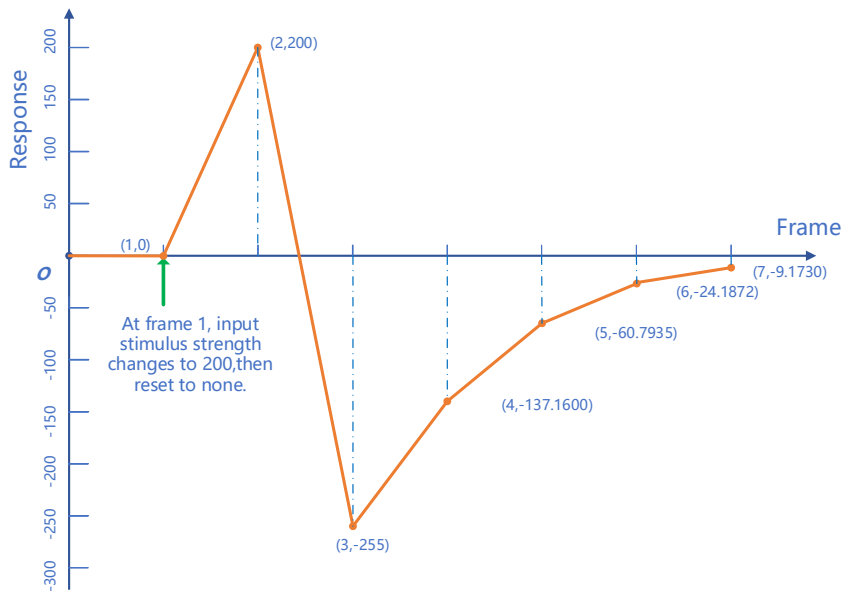

(b) $\operatorname{Pt}(\mathrm{x}, \mathrm{y})-\operatorname{Lt}(\mathrm{x}, \mathrm{y})$

Fig. 1. (a)Refractoriness schematic diagram. The orange curve shows the change of membrane potential. Depolarization and repolarization are represented by dashed line with arrow. ARP corresponds to depolarization and part of repolarization while RRP is covered by hyperpolarization.(b) shows the curve of $P_{t}(x, y)-L_{t}(x, y)$ when a single stimulus is applied at $1^{s t}$ frame, which resembles the real membrane potential curve during RP.

tecting collision when inbound objects are ultra-fast; it also contributes to suppressing the LGMD when collision is not detected, reducing false alerts. And in a subtle manner, it equips previous works with ability of eliminating influence of low level noises to some extent. Thus, this research demonstrates RP can be promising for enhancing LGMD model's reliability and stability in the future works.

The structure of the remaining is organised as follows: in section II, most relative works are briefly reviewed. Section III elucidates the model in detail as well as parameter settings. Section IV explains materials that have been utilized in the systemically comparative experiments, followed by section $\mathrm{V}$ shows recorded outcomes of experimental evaluation. Section VI concludes this study with discussion.

\section{RELATED WORKS}

In this section, we introduce the most related works in the subsection A) bio-plausible collision perception models inspired by locusts' visual system, and B) modelling of refractory period.

\section{A. Bio-Plausible Collision Perception Models}

Thanks to millions of years evolution, invertebrates have been provided with reliable and robust visual systems that allow them to survive clustered environments and predating enemies coming out of nowhere at surprisingly high speed.

Though LGMD is widely researched in drosophila's and crab's vision systems [8], [9], most literature take a locust that flies as typical example for collision perception. LGMD1, identified in the lobula layer inside its visual pathways, has firstly been discovered as movement detectors in [10], then been recognized to produce the most frequent spikes to objects on an approaching course while respond weakly to receding or translating objects [11]. Subsequently a neural network composed of four groups of neurons, namely the photoreceptor layer ( $P$ layer), the excitation layer ( $E$ layer), the inhibition layer ( $I$ layer) and the summation layer $(S$ layer), was proposed by Rind together with Bramwell [12], based on which mathematically modelling of the LGMD1 was further accomplished by Gabbiani et al. focused on angular velocity in [13], and Yue et al. in [14] by adding a novel group layer ( $G$ layer), which concluded, through offline and online experiments, that the classic LGMD model equipped the minirobot, Khepera robot (K-Team, Switzerland) at the speed of $32 \mathrm{~mm} / \mathrm{s}$, with the ability of cruising autonomously and free of collision in real time inside the experimental arena. The model was then similarly converted to an embedded visual system strategy. Hu et al. applied this method to a microrobot, proving its high precision and reliability in collision detection by running the micro-robot at various velocities from $1.5 \mathrm{~cm} / \mathrm{s}$ up to $17 \mathrm{~cm} / \mathrm{s}$ in a arena with several sets of densities of obstacles [3]. Recently, by choosing a larger convolutional mask, Zhao et al. expanded LGMD1 model's application field to small quadcoptors, equipping them with ability of sensing impending collision [15]. To enhance the precision and expand the application of visual-based collision detection neural networks, an integrated model of LGMD1 and translating sensitive neural network (TSNN), which consists of four directionally sensitive neurons based correlated elementary movement detectors (EMDs), was proposed by Yue et al. in [16]. Additionally, a specialized decision-making mechanism coordinates with the integrated model, achieving more reliable and robust responses to clustered background.

As an interneuron of LGMD1, LGMD2, though shares the same features of responding more strongly to looming objects, has its distinct characterization. Having been found to mature early in juvenile locusts, this group of neurons has been investigated to show most preference to dark incoming objects 
within a bright background, which resembles their surviving environment as young individuals, than a dark background [17]. Regardless of its significance for locusts that mainly live on the ground, only a few studies have been published. It is only after 2015 that computationally modelling of LGMD2 was completed by $\mathrm{Fu}$ and Yue [18], realising its selectivity to light-to-dark luminance changing by introducing a novel mechanism of ON \& OFF parallel channels, discriminating luminance increment and decrement. More specifically, in the ON channels, which are rigorously sieved, inhibitions and temporally delayed excitations are produced while in the OFF channels, direct excitation and temporally delayed inhibition are generated [19]. Both offline tests and real-time experiments on the aforementioned micro-robot demonstrated feasibility and robustness of this creative algorithm [20].

Though the aforementioned models have made remarkable contributions to collision detection systems based on visual cues and in turn inspired exploration on coordination mechanism of interneurons, their performance could drop sharply under certain circumstances, such as the velocity of an object on a colliding trajectory is relatively high, up to $9.6 \mathrm{~m} / \mathrm{s}$ and the input signal (image) is noise-contaminated.

\section{B. Modelling for Refractoriness}

Refractoriness has been comprehensively noticed and investigated as a spontaneous mechanism inside animal neural systems for a considerably long time. As far back as 1970s, Ruzi introduced ARP into a model of RATEN, randomthreshold neuron-like element networks proposed by Amari, to further improve its stability in [21], presenting positive impact of integrating ARP with existing models.

As an issue of concern, after abundant efforts, mathematically modelling has made extraordinary progresses. In the attempts to reveal the code of information transmission between spiking neurons, several sorts of stochastic processes have been included to estimate refractoriness [22]. In [23], a simplified Poisson process, which is utilized to estimate the characteristics of refractoriness is further adopted to suit other input inter-pulse intervals (IPIs). Another study from Schaette et al. proposed a renewal process with a recovery function to describe neural refractoriness. Though comparative experiment, this estimation shows close match between the observed locust auditory receptor neurons spike trains, presenting the significance of refractoriness to artificial neural networks [24]. Though Song et al. touched the combination of refractoriness and flies' photoreceptor, and elucidated what role RP plays in the encoding of graded neural responses, exploiting it for collision detection has not been considered [25].

By building computational models of RP, these works numerically contributed to our understanding the how underlying neuron circuits incorporate and the mechanism of how non-linear calculation conducts inside the neuronal pathways. However, infrequently are they considered to be integrated with collision perception visual models to further improve their accuracy and robustness.

\section{Formulation of the Method}

In this section, we present the strategy of the proposed RP mechanism fused with LGMD model with formulations as well as parameter settings.

Sharing most structures of classic LGMD neural network in [14], the first layer, $P$ layer of our proposed model is composed photoreceptors that are sensitive to brightness changing, and it is calculated by

$$
P_{t}(x, y)=B_{t}(x, y)-B_{t-1}(x, y)
$$

where $B_{t}(x, y)$ and $B_{t-1}(x, y)$ respectively corresponds to the luminance value of pixel $(x, y)$ at the moment $t$ and $t-1$. The core of our proposed model lays in the $L$ layer, which is placed between $P$ and $E$ layer to determine whether the output of $P$ layer can be passed to its connected $E$ layer. $L$ layer, a 2-D matrix of the size of $P$ layer, consists of local thresholds, and is defined by both a decay indicator $i_{t}$ and the value of corresponding $P$ layer cell:

$$
L_{t}(x, y)=\left\{\begin{array}{l}
L_{\text {max }}, \quad \text { if }\left|P_{t}(x, y)\right|>L_{t-1}(x, y) \\
L_{\text {max }} \cdot \alpha \cdot\left(1+e^{i_{t}(x, y)}\right)^{-1}, \quad \text { otherwise }
\end{array}\right.
$$

where $\alpha$ is a coefficient to scale the decay function. While $L_{\max }$ is the upper boundary that $P_{t}(x, y)$ can reach. More specifically, if $P_{t}(x, y)$ manages to exceed $L_{t-1}(x, y)$, the consecutive local threshold $L_{t}(x, y)$ rises to $L_{\max }$; otherwise $L_{t}(x, y)$ decays exponentially and is updated with $i$, which is determined by the following equation:

$$
i_{t}= \begin{cases}1, & \text { if } \quad P_{t}(x, y)>L_{t-1}(x, y) \text { or } \\ & i_{t-1} \geqslant T_{\text {decay }} \\ i_{t-1}+1, & \text { otherwise }\end{cases}
$$

where the $T_{\text {decay }}$ is a determining constant for how many frames that a local threshold decays according to equation (1) case otherwise. Subsequently, while values of activated photoreceptor cells are inherited by cells in the $E$ layer at corresponding point, these values, with one image frame delay, flows into $I$ layer as well, where inhibition is passed, elucidated by

$$
\begin{gathered}
I_{t}(x, y)=\sum_{i} \sum_{j} P_{t-1}(x+i, y+j) \cdot w_{I}(i, j) \\
w_{I}=\left[\begin{array}{ccc}
0.125 & 0.25 & 0.125 \\
0.25 & 0 & 0.25 \\
0.125 & 0.25 & 0.125
\end{array}\right]
\end{gathered}
$$

where $I_{t}(x, y)$ represents the inhibition of the cell positioned at $(x, y)$ at $t$ moment. $w_{I}(i, j)$ indicates a $3 \times 3$ local convolutional kernel representing local inhibition weight, which allows local inhibition to pass through to its neighbouring cells, thus $i$ and $j$ shall not concurrently equal to zero. The following layer is $S$ layer, where excitation transmitted from $E$ layer and inhibition from $I$ layer is summed by the following equation:

$$
S_{t}(x, y)=E_{t}(x, y)-I_{t}(x, y) \cdot W_{I}
$$




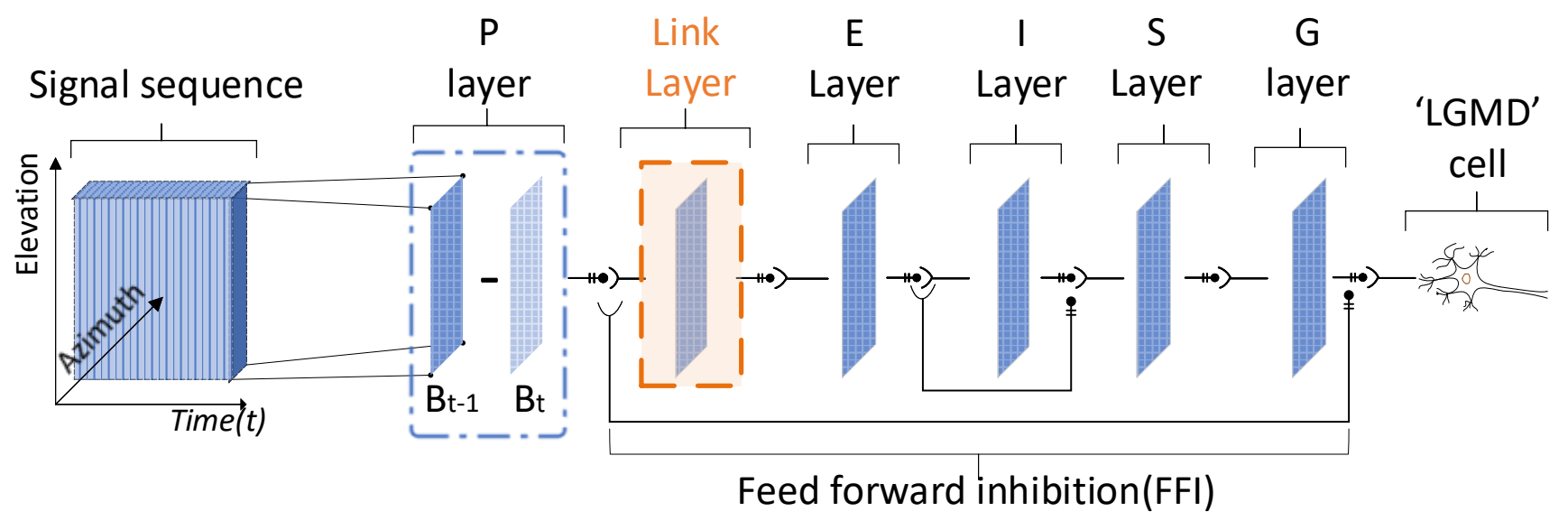

Fig. 2. Schematic of LGMD neural network with the Link layer. The blue cuboid on the left side represents motion stimuli. Within $P$ layer, image frame at $t$ time subtracts its previous one. The output of $P$ is then conveyed to $L$ layer, and so on.

where $W_{I}$ is a weight parameter constant. Here, outcomes of $S$ cells, instead of being gathered immediately by the $L G M D$ neuron, are injected into a group layer ( $G$ layer) to further enhance the developing edges derived from complex stimuli via a specialized convolutional operation, which is given by

$$
\begin{gathered}
{[C e]_{t}=[S]_{t} \otimes\left[w_{e}\right]} \\
w_{e}=\frac{1}{9} \times\left[\begin{array}{lll}
1 & 1 & 1 \\
1 & 1 & 1 \\
1 & 1 & 1
\end{array}\right]
\end{gathered}
$$

where $\otimes$ is defined as a convolution operator, and $w_{e}$ accordingly is the convolutional mask. The output $C e_{t}$ is then forwarded to $G$ layer. Please note that $C e_{t}$ shall be partially discarded to match the magnitude of $S_{t}$. Capable of providing stronger input to the consecutive $L G M D$ cell, $G$ layer is explicit as follows:

firstly, raw data is calculated by

$$
g_{t}=w^{-1}\left([S]_{t} *[C e]_{t}\right)
$$

where $w^{-1}$ is a scalar for the Hadamard product of $[S]_{t}$ and $[C e]_{t}$. The scalar is updated every frame by the following equation:

$$
w=\Delta c+\max \left(a b s[C e]_{t}\right) \cdot C_{w}^{-1}
$$

within which $\Delta c$ is a small positive real number while $C_{w}^{-1}$ is a constant, and $\max \left(a b s[C e]_{t}\right)$ extracts the maximum value within the matrix $C e_{t}$.

Secondly, the raw $g_{t}$ is further selected by

$$
G_{t}(x, y)= \begin{cases}g_{t}(x, y), & g_{t}(x, y) \cdot C_{f a} \geqslant T_{f a} \\ 0, & \text { otherwise }\end{cases}
$$

where $C_{f a}$ denotes the fading coefficient that belongs to ( 0 , 1 ), and $T_{f a}$ is the fading threshold. After the $G$ layer, not only the edge information collected from approaching motion is further strengthened, but also the excitation collected from clustered background is cancelled to a certain degree. The product $G_{t}$ subsequently is conveyed to the $L G M D$ neuron to be calculated by following equations divided into two steps:

$$
K_{t}=\sum_{x} \sum_{y} a b s\left(G_{t}(x, y)\right)
$$

where firstly the summation of membrane potential, $K_{t}$, is calculated by sum every absolute value of pixel in $G$ layer. Secondly the aforementioned summation is normalized utilizing a sigmoid function stated below:

$$
L G M D_{t}=\left(1+e^{-K_{t} \cdot n_{\text {cell }}^{-1}}\right)^{-1}
$$

where $n_{\text {cell }}$ counts photoreceptors on the retina. Once the consequence of $L G M D$ neuron, which is limited in the range $[0.5,1]$, exceeds the stationary threshold $T_{l g m d}$, one spike is fired:

$$
\text { spike }= \begin{cases}1, & \text { if } L G M D_{t}>T_{\text {lgmd }} \\ 0, & \text { otherwise }\end{cases}
$$

Under physical circumstances, turning provokes enormous luminance change that triggers the LGMD model in a predominant manner. Hence, the Feed Forward Inhibition (FFI) mechanism is introduced together with lateral inhibition to suppress rapid luminance change caused by turning. It functions as the following equations:

$$
F_{t}=n_{c e l l}^{-1} \cdot \sum_{x} \sum_{y}\left|P_{t-1}(x, y)\right|+\sum_{i}^{n_{a}}\left(\alpha_{t-1}^{F} \cdot F_{t-i}\right)
$$

where $\alpha_{t-1}^{F}$ denotes a coefficient which belongs to $(0,1)$, weighing the impact of previous $F_{t-i}$. Then the result $F_{t}$ is compared with a threshold, $T_{f f i}$, which is updated to time by

$$
T_{f f i_{t}}=T_{O}+\alpha_{f f i} \cdot T_{f f i_{t-1}}
$$

where $T_{O}$ is the original value of $T_{f f i}$ and $\alpha_{f f i}$ is a scalar.

Parameter settings refers to Table I.

Compared with those methods that require more computational power such as objective detection and large-scale driving 
TABLE I

The Parameters SetTing

\begin{tabular}{llllll}
\hline \multicolumn{6}{c}{ Parameter: Name, Value (Val) } \\
\hline Name & Val & Name & Val & Name & Val \\
\hline$L_{\max }$ & 255 & $\Delta_{C}$ & 0.01 & $T_{O}$ & 7.5 \\
$\alpha$ & 2 & $C_{w}$ & 4 & $\alpha_{f f i}$ & 0.02 \\
$T_{\text {decay }}$ & 7 & $C_{f a}$ & 0.5 & $T_{\text {lgmd }}$ & 0.7 \\
$W_{I}$ & 0.3 & $T_{f a}$ & 15 & $i_{0}$ & 1 \\
\hline
\end{tabular}

scene reconstruction [26], this LGMD1-based is acceptable in terms of accuracy and robustness while considerably low in algorithm complexity, which retains the potential for being further embedded into low-energy consuming hardware platforms.

\section{Materials}

Previously, while exploring performance of LGMD neural networks, researchers utilize video signals, where the velocity of objects is usually as low as no more than $1 \mathrm{~m} / \mathrm{s}$, as their offline experimental materials. As for the real-time tests carried on a micro-robot, the velocity is usually measured in centimetres. Thus, in this study to explore how refractoriness affects the performance of LGMD model when faced with ultra-fast objects on a colliding course, we utilize videos recorded under experimental circumstances, where the velocity of the incomings is more than $5 \mathrm{~m} / \mathrm{s}$, up to around 9.6 $\mathrm{m} / \mathrm{s}$. As the RP is fairly short period of time [27], aiming at mimicking the actual duration of RP, a high-speed dynamic camera, GoPro8, is used to sample the processes of a black ping-pang ball approaching and hitting its lens at sampling rate of $240 \mathrm{~Hz}$, that is to say, capture one frame every 4.1667 millisecond, 8 times as quick compared to $30 \mathrm{~Hz}$ which is the sampling rate previous works adapts. In this way, we approach closer to the real duration of RP.

In subsection V-B, 20 video clips shot on GoPro8 are included. Individual clip at sampling rate (or frame rate) 240 $H z$ is resized to $320 \times 180$ from $1920 \times 1080$, then decimated to sampling rate of $30 \mathrm{~Hz}$ and $24 \mathrm{~Hz}$. To realise this, designated frames from original videos are extracted according to the targeting frame rate. Taking $120 \mathrm{~Hz}$ frame rate for example, every $\frac{240 \mathrm{~Hz}}{120 \mathrm{~Hz}}$ frames makes up one 'frame set', within which every $1^{\text {st }}$ frame is used to form new clips. Then frames of the same amount that cover the whole approaching course are intercepted to be input as signals.

In subsection $\mathrm{V}-\mathrm{C}$, the same 20 clips are then polluted by Gaussian noise or Salt-Pepper noise at different Signal-tonoise ratio (SNR).

A computer-generated simulative video is used as well, where a black square at the heart begins to grow at $15^{\text {th }}$ frame and pauses at $36^{\text {th }}$ frame within grey background.

\section{EXPERIMENTAL EVALUATION}

In this section, we step-by-step illustrate the experimental results, divided into 3 steps: a) feasibility of the proposed model with RP mechanism, b) effectiveness of proposed model against ultra-fast inbound objects and c) performance against noise-polluted signals.

\section{A. Feasibility of RP}

Firstly we test the model with aforementioned computergenerated simulative signal and GoPro-captured video to validate the feasibility of integrating RP mechanism with classic LGMD model. Results are illustrated by Fig. 3 and Fig. 4 clearly. The orange curves represent the normalized responses of LGMD neuron (Eq. 13) handled by $L$ layer and RP mechanism in our proposed model, while the blue ones show output of classic LGMD model. The green horizontal lines are $T_{l g m d}$ we set to recognize collision. From the outcomes, it can be noticed that RP mechanism causes LGMD observes and spikes for collision with one or several frames delay, which narrows down the range of collision.

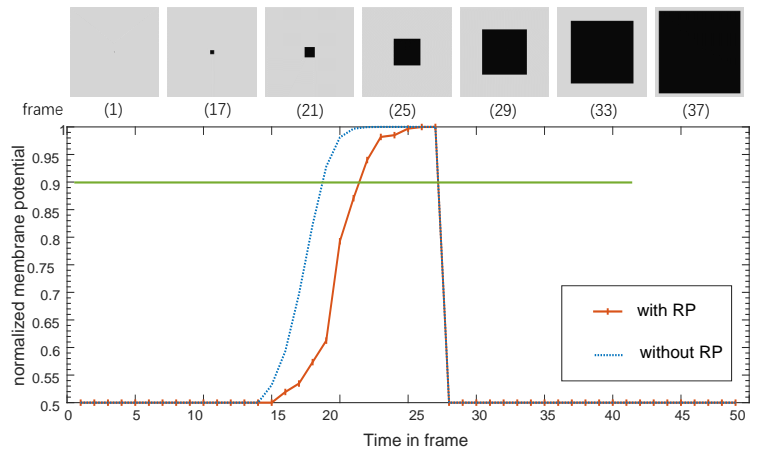

Fig. 3. Examples of snapshots of the computer-generated video with corresponding frame number tagged below. With the black square grows, the responses gets more intensive and trespasses the $T_{l g m d}$ (set to 0.9 for simulative signals) until reaches the upper boundary. Orange curve and blue dashed curve clearly show that both models succeed in extracting the motion pattern of approaching.

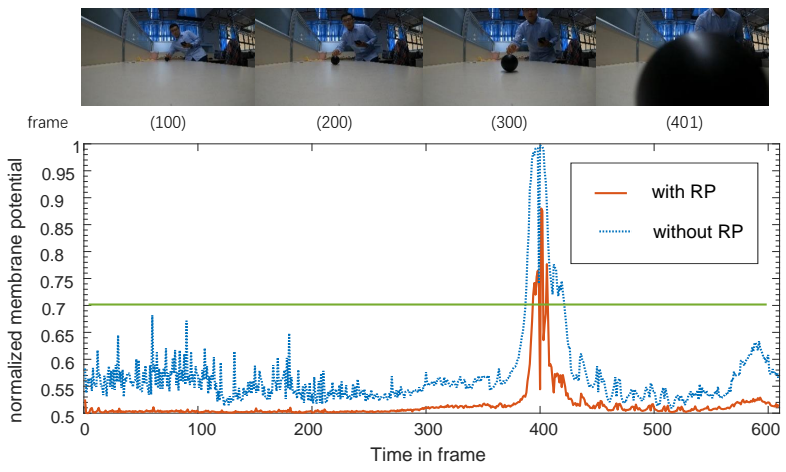

Fig. 4. Snapshots of the recorded signal at $240 \mathrm{~Hz}$ with frame number tagged below. At an average velocity of $0.8727 \mathrm{~m} / \mathrm{s}$ and initial velocity of $0 \mathrm{~m} / \mathrm{s}$, the black ball starts to roll to camera lens from $1 \mathrm{~m}$ away at $122^{\text {nd }}$ frame, and hits it at $401^{\text {st }}$ frame, followed by frames where the ball goes backwards. 0.7 is set as $T_{\text {lgmd }}$ in Eq.14 for real-world signals. 


\section{B. Effectiveness against Ultra-Fast Objects}

Here when 'ultra-fast' is talked about, we not only refer to real-world velocity, but also mean angular velocity from the perspective of the LGMD neural network, which triggers the LGMD neuron falsely and repeatedly. By lowering sampling rate of the 20 GoPro8-shooting videos, continuous motion is further discretized in time dimension, thus objects seem to move faster if the lowered sampling rate mapped again from low to higher, due to comparatively more immense difference between two frames.

From the consequences of comparative experiments calculated by Eq.13, it can be noticed that when signals are sampled at $240 \mathrm{~Hz}$, both models succeed in recognizing impending collision during the collision zone (between by 2 red dashed lines), but without RP, LGMD neuron produces spikes several frames earlier.

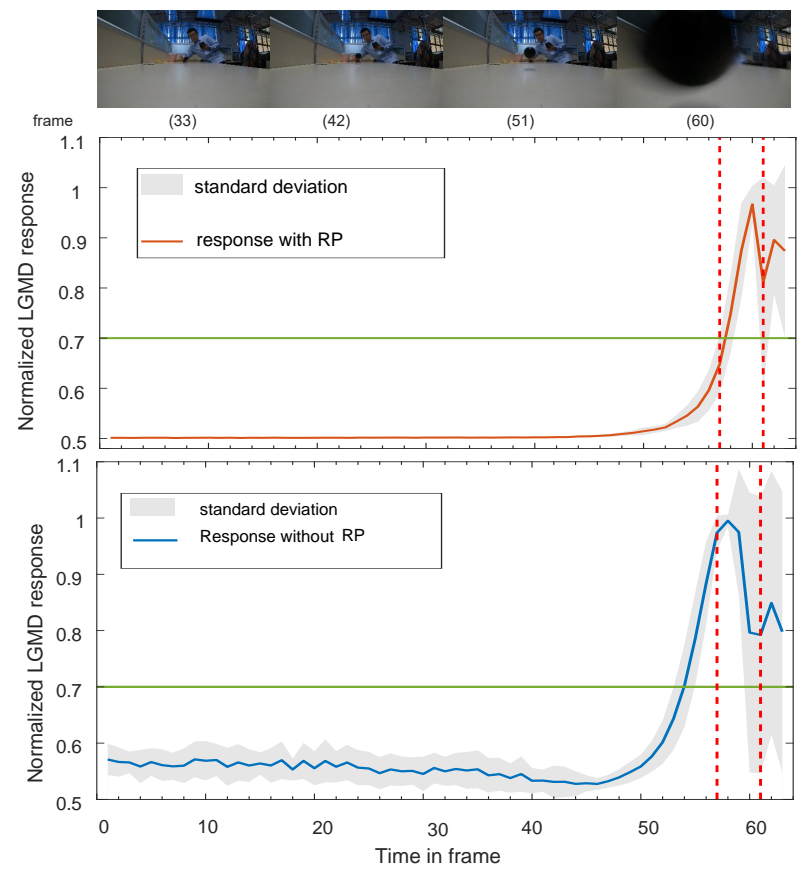

Fig. 5. Example snapshots of one of video signals and statistical results below frame number tags. $T_{l g m d}$ illustrated by horizontal green line. Between two red dashed lines are collision zone. The orange curve and blue curve represent the average LGMD membrane potential at every corresponding frame or moment in 20 video clips respectively. Light grey area covers the standard deviation of all signals at a certain frame. Collision is reported by both models. Orange curve shows collision is extracted while blue one reports collision several frames earlier. The orange curve also shows stationary property before collision, compared with the blue one.

For low sampling rate signals, we test both model with 30 $\mathrm{Hz}$ as well as $24 \mathrm{~Hz}$. Though classic LGMD without RP mechanism is activated during collision zone, it fires spikes when the objects are still far away from the camera lens, meaning false alert, while our proposed model with RP notifies the right collision.

It is also worth paying attention to the results outside the collision zone, where LGMD is not supposed to fire, our

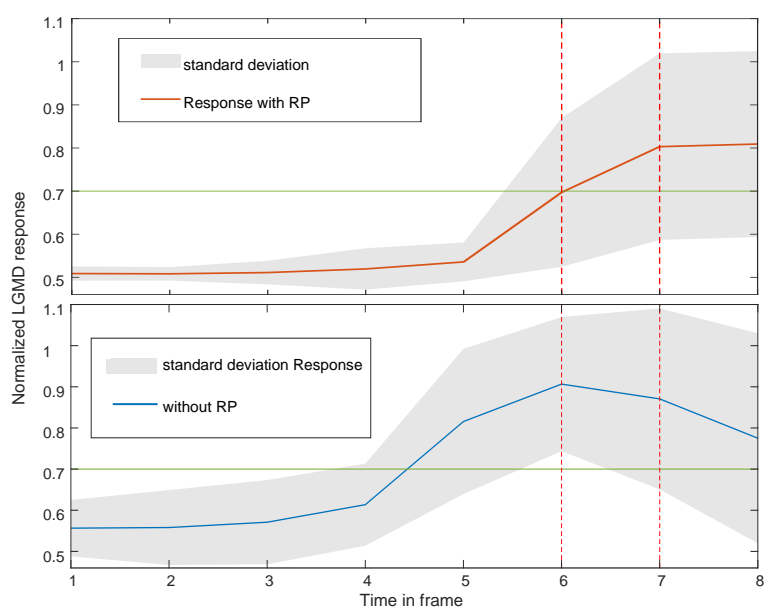

Fig. 6. Statistical results of signals at sampling rate $30 \mathrm{~Hz}$

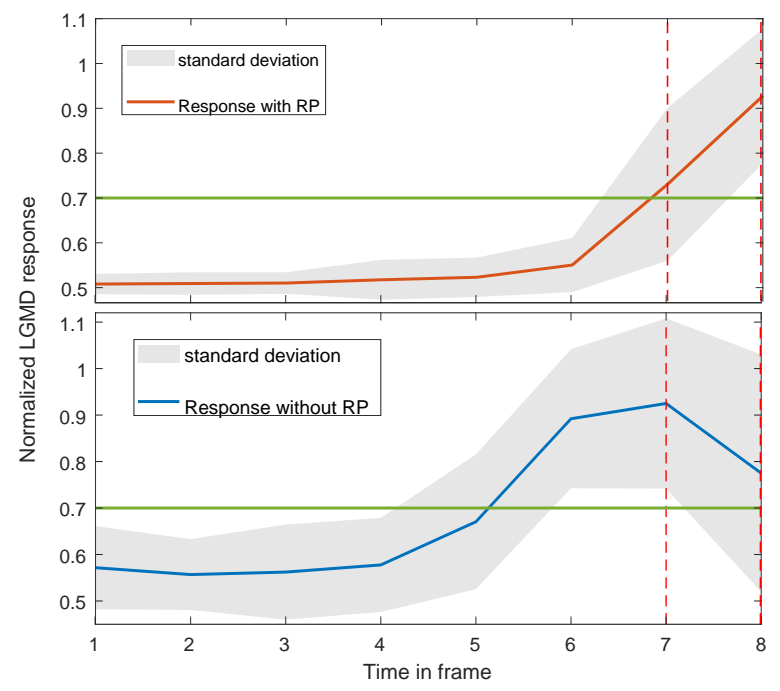

Fig. 7. Statistical results of signals at sampling rate $24 \mathrm{~Hz}$.

model demonstrates effectiveness on silencing the neuron in Fig.5, and in Fig.6 and Fig.7, RP also shows influence on weakening the fluctuation but not that obvious compared with high sampling rate signals. This, to some extent, suggests that useless and small edge expansion patterns may have been filtered while continuous expansion reserved for further collision perception.

\section{Performance against Noisy-Polluted Signals}

Subsequently, PR is tested for noise resistance ability, with the same $240 \mathrm{~Hz}$ signals used in subsection V-B polluted by either Gaussian noise or Salt-Pepper noise. Some of our experimental results show delightful efficacy on resisting the low-strength noise such as Gaussian noise (Fig.8). Although compared to classic model, RP mechanism helps to purify contaminated signals, it is still challenging to extract collision 


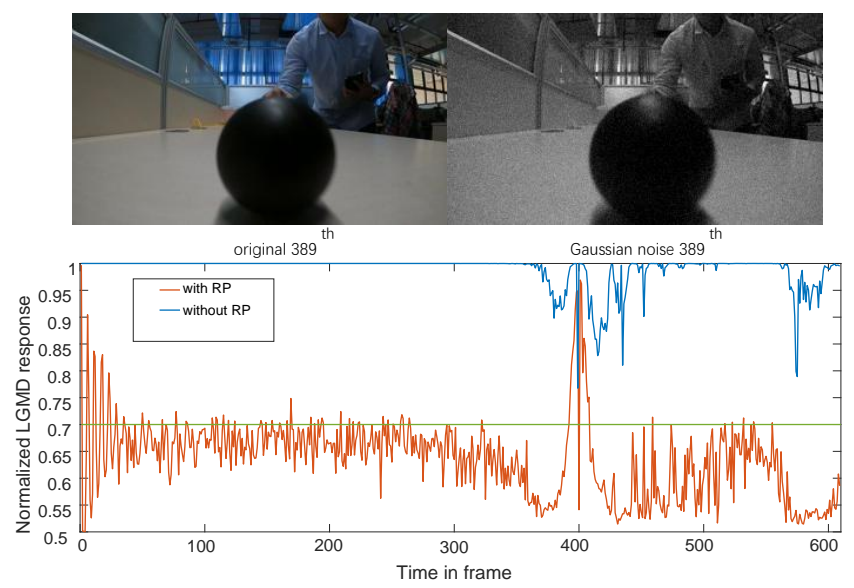

Fig. 8. Snapshots of $389^{\text {th }}$ frame from original video and Gaussian-noisecontaminated video. The orange curve represents LGMD membrane potential (referring to Eq.13) with RP mechanism, comparatively blue one without RP. While most of the blue curve stays at 1, orange curve can be easily distinguished for the peak at $401^{\text {st }}$ frame with violent fluctuation within first 40 frames.

and approaching motion pattern when SNR drops as shown in Fig.9.

\section{CONCLUSION AND DisCUSSION}

While cruising in complex real world, locusts amazingly demonstrate their ability to avoid impending collision and incoming objects at high speed, including those in complex environments as well as the homologous. In this paper, we propose an integrated model of LGMD and refractoriness mechanism based on prior knowledges of locusts' visual system. Compared the original LGMD1 model, our method shows advantages on three aspects: 1) feasibility of recognizing ultrafast objects on a colliding trajectory, the velocity of which reaches up to $9.6 \mathrm{~m} / \mathrm{s} ; 2$ ) stability outside the collision zone where only useless edge expansion are filtered; 3 ) in a subtle manner,it also improve the classic LGMD model's performance when input signals are impure, especially are low-density Gaussian or Salt-Pepper noise polluted. Hence, it is shown that RP is promising in modelling the collision perception neural networks based on visual cues to handle complex signals that resemble what locusts are seeing in a gliding scene in the future.

Though our simplified model basically realises characterization of RP mechanism together with the classic LGMD model, compared to modelling RP based on stochastic processes, it remains to be further accomplished in terms of algorithm of RP. An question occurs then: is it possible to make LGMD model noise-resisting in the meanwhile stays low demanding in terms of computational power? It is believed an enhanced modelling of RP integrated with LGMD models, including those focused on angular velocity and those combining multiple neurons, could be a solution for balancing noising-cancelling and low algorithm complexity.

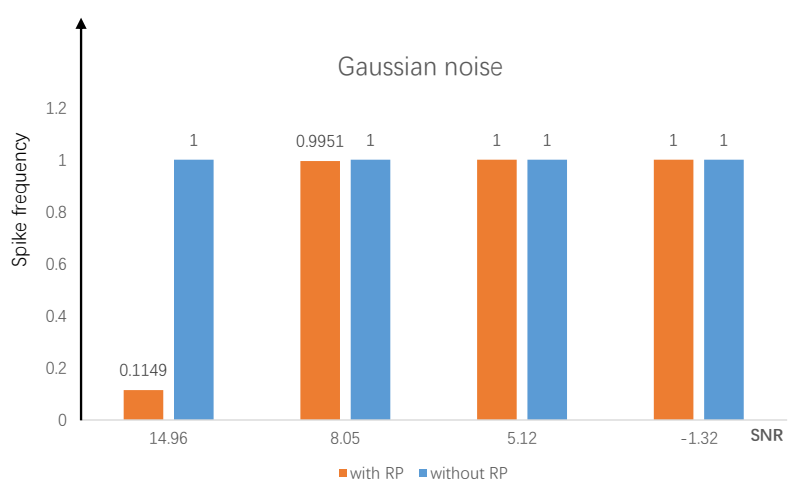

(a) Gaussian noise

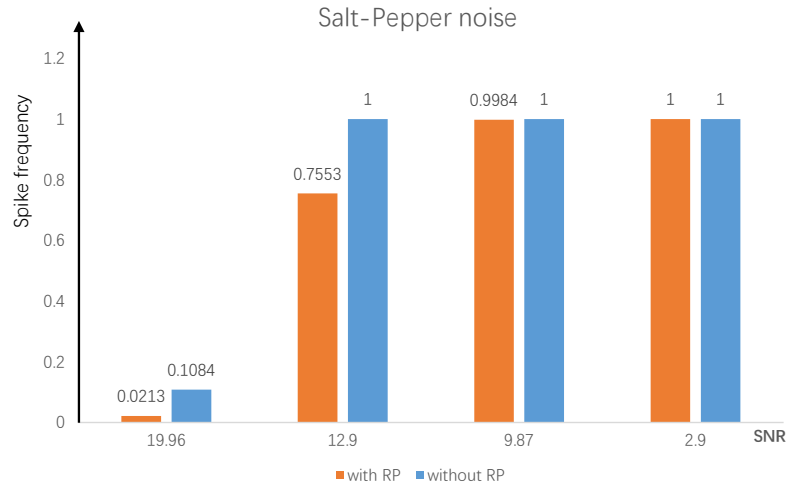

(b) Salt-Pepper noise

Fig. 9. Here, spike frequency is the product of amount of spike divided by amount of frames. (a) Orange bars illustrate that, spike frequency with RP mechanism interfering is low at SNR equalling 14.96, while blue ones show that LGMD is compromised by Gaussian noise. (b) Against low level SaltPepper noise, both models work properly and observe collision successfully. Comparatively, RP mechanism improves LGMD in terms of noise resistance.

\section{ACKNOWLEDGEMENT}

This research has received funding from the European Union's Horizon 2020 research and innovation programme under the Marie Sklodowska-Curie grant agreement No 778602 ULTRACEPT, and the China Postdoctoral Science Foundation Grant 2020M682651.

\section{REFERENCES}

[1] F. C. Rind and P. J. Simmons, "Seeing what is coming: building collision-sensitive neurones," Trends in Neurosciences, vol. 22, no. 5, pp. 215-220, 1999.

[2] J. Byrne and C. J. Taylor, "Expansion segmentation for visual collision detection and estimation," in 2009 IEEE International Conference on Robotics and Automation, 2009, pp. 875-882.

[3] C. Hu, F. Arvin, and S. Yue, "Development of a bio-inspired vision system for mobile micro-robots," in 4th International Conference on Development and Learning and on Epigenetic Robotics, 2014, pp. 8186.

[4] J. Pyo, J. Bang, and Y. Jeong, "Front collision warning based on vehicle detection using cnn," in 2016 International SoC Design Conference (ISOCC), 2016, pp. 163-164.

[5] J. Lai, L. Mejias, and J. J. Ford, "Airborne vision-based collisiondetection system," Journal of Field Robotics, vol. 28, no. 2, pp. 137-157, 2011.

[6] S. Nedevschi, A. Vatavu, F. Oniga, and M. M. Meinecke, "Forward collision detection using a stereo vision system," 2008, pp. 115-122. 
[7] E. Hamel and R. Labib, "Modeling biological refractory peridos and synaptic depression in an artificial neuron," Biomedical Physics \& Engineering Express, vol. 5, no. 2, pp. 25-38, 2019.

[8] L. Li, Z. Zhang, and J. Lu, "Artificial fly visual joint perception neural network inspired by multiple-regional collision detection," Neural Networks, vol. 135, pp. 13-28, 2021.

[9] J. Carbone, A. Yabo, and D. Oliva, "Characterization and modelling of looming-sensitive neurons in the crab neohelice," Journal of Comparative Physiology A, vol. 204, pp. 487-503, 2018.

[10] M. O' Shea and J. L. Williams, "The anatomy and output connection of a locust visual interneurone; the lobular giant movement detector (lgmd) neurone," Journal of comparative physiology, vol. 91, pp. 257266, 1974.

[11] F. C. Rind, S. Wernitzing, P. Pölt, A. Zankel, D. Gütl, J. Sztarker, and G. Leitinger, "Two identified looming detectors in the locust: ubiquitous lateral connections among their inputs contribute to selective responses to looming objects," Scientific Reports, vol. 6, no. 1, p. 35525, 2016.

[12] F. C. Rind and D. I. Bramwell, "Neural network based on the input organization of an identified neuron signaling impending collision," Journal of Neurophysiology, vol. 75, no. 3, pp. 967-985, 1996.

[13] F. Gabbiani, H. G. Krapp, C. Koch, and G. Laurent, "Multiplicative computation in a visual neuron sensitive to looming," Nature, vol. 420, pp. 320-324, 2002.

[14] S. Yue and F. C. Rind, "Collision detection in complex dynamic scenes using an lgmd-based visual neural network with feature enhancement," IEEE Transactions on Neural Networks, vol. 17, no. 3, pp. 705-716, 2006.

[15] J. Zhao, C. Hu, C. Zhang, Z. Wang, and S. Yue, "A bio-inspired collision detector for small quadcopter," in 2018 International Joint Conference on Neural Networks (IJCNN), 2018, pp. 1-7.

[16] S. Yue and F. C. Rind, "Visual motion pattern extraction and fusion for collision detection in complex dynamic scenes," Computer Vision and Image Understanding, vol. 104, no. 1, pp. 48-60, 2006.

[17] J. Sztarker and F. C. Rind, "A look into the cockpit of the developing locust: looming detectors and predator avoidance," Developmental neurobiology, vol. 74, no. 11, pp. 1078-1095, 2014.

[18] Q. Fu and S. Yue, "Modelling lgmd2 visual neuron system," in 2015 IEEE 25th International Workshop on Machine Learning for Signal Processing (MLSP), 2015, pp. 1-6.

[19] Q. Fu, C. Hu, T. Liu, and S. Yue, "Collision selective lgmds neuron models research benefits from a vision-based autonomous micro robot," in 2017 IEEE/RSJ International Conference on Intelligent Robots and Systems (IROS), 2017, pp. 3996-4002.

[20] Q. Fu, C. Hu, J. Peng, F. C. Rind, and S. Yue, "A robust collision perception visual neural network with specific selectivity to darker objects," IEEE Transactions on Cybernetics, vol. 50, no. 12, pp. 5074 5088,2020

[21] J. G. Ruiz, "Random threshold element networks with absolute refractory period," Proceedings of the IEEE, vol. 64, no. 8, pp. 1257-1259, 1976.

[22] D. Hampel and P. Lansky, "On the estimation of refractory period," Journal of neuroscience methods, vol. 171, no. 2, pp. 288-295, 2008

[23] X. L. Hu and Y. T. Zhang, "Effects of refractoriness on the statistics of the post-membrane output ipis," in Proceedings of the 22nd Annual International Conference of the IEEE Engineering in Medicine and Biology Society (Cat. No.00CH37143), vol. 1, 2000, pp. 589-591.

[24] R. Schaette, T. Gollisch, and A. V. M. Herz, "Spike-train variability of auditory neurons in vivo: Dynamic responses follow predictions from constant stimuli," Journal of Neurophysiology, vol. 93, no. 6, pp. 32703281,2005

[25] Z. Song, Y. Zhou, and M. Juusola, "Modeling elucidates how refractory period can provide profound nonlinear gain control to graded potential neurons," Physiological reports, vol. 5, no. 11, 2017.

[26] V. Usenko, J. Engel, J. Stückler, and D. Cremers, "Reconstructing streetscenes in real-time from a driving car," 2015, pp. 607-614.

[27] M. J. Berry and M. Meister, "Refractoriness and neural precision," Journal of Neuroscience, vol. 18, no. 6, pp. 2200-2211, 1998. 